Ebisu Ebisu

Études japonaises Études japonaises

50 | automne-hiver 2013

Création et valeurs dans le Japon moderne

\title{
Valorisation boursière comparée des entreprises familiales et non familiales au Japon
}

日本の同族・非同族会社汶対寸る株式市場評価

Market Valuation of Family and Non-Family Businesses in Japan

Bruno Amann, Jacques Jaussaud et Sophie Nivoix

\section{OpenEdition}

Journals

Édition électronique

URL : http://journals.openedition.org/ebisu/1088

DOI : 10.4000/ebisu. 1088

ISSN : 2189-1893

Éditeur :

Institut français de recherche sur le Japon (UMIFRE 19 MAEE-CNRS), Maison franco-japonaise

Édition imprimée

Date de publication : 1 octobre 2013

Pagination : 7-25

ISSN : 1340-3656

Référence électronique

Bruno Amann, Jacques Jaussaud et Sophie Nivoix, « Valorisation boursière comparée des entreprises familiales et non familiales au Japon », Ebisu [En ligne], 50 | automne-hiver 2013, mis en ligne le 21 juin 2014, consulté le 01 mai 2019. URL : http://journals.openedition.org/ebisu/1088 ; DOI : 10.4000/ ebisu. 1088 


\section{Valorisation boursière comparée des entreprises familiales et non familiales au Japon}

Bruno AMANN, Jacques JAUSSAUD, Sophie NIVOIX*

Il y a quelques années encore, le domaine des entreprises familiales (famirī kigyō ファミリー企業) n'était pas un domaine de recherche. Il restait marqué tant par une certaine littérature romanesque que par un désintérêt des chercheurs en sciences sociales. Depuis quelques années, les travaux sur les entreprises familiales constituent cependant un mainstream, et ce champ de recherches est aujourd'hui entré dans son " adolescence " (Gedajlovic et al. 2011). Comme dans tout domaine, les questions posées par la recherche sont multiples. Certaines demeurent délaissées.

La plupart des études sur les sociétés cotées n'opèrent pas de distinction entre les entreprises selon la nature, familiale ou non, de leur actionnariat ${ }^{1}$. La grande majorité des analyses portant sur les entreprises familiales se focalise en outre sur d'autres aspects que la valorisation boursière. L'essentiel des recherches liées à la valorisation boursière, enfin, ne s’intéresse pas aux entreprises familiales en tant que telles.

Le présent travail souhaiterait par conséquent faire quelques propositions afin de tenter de combler ce manque. C'est pourquoi, dans le contexte particulier des entreprises et du marché financier japonais, auxquels nous

* Bruno Amann, université Toulouse 3 ; Jacques Jaussaud, université de Pau et des Pays de l'Adour ; Sophie Nivoix, université de Poitiers.

1. D'une manière générale, sur les effets de l'implication de la famille dans l'entreprise, voir Ayranci 2010. 
avons consacré déjà un certain nombre de travaux, nous avons souhaité étudier la valorisation des entreprises familiales par le marché des actions. En nous appuyant sur la méthode particulière du pairage ${ }^{2}$, notre étude vise à fournir une comparaison solide entre les entreprises familiales et non familiales au Japon, afin de déceler si des écarts existent en matière de valorisation boursière. Différents ratios seront utilisés dans cette démarche.

Le cas du Japon est particulièrement intéressant à cet égard, puisque le pays possède un long passé d'entrepreneuriat familial, qui remonte bien avant l'ouverture de l'archipel aux Occidentaux au XIX ${ }^{e}$ siècle. Certaines entreprises japonaises compteraient ainsi parmi les plus anciennes au monde, avec par exemple Kongō Gumi 金剛組 fondée en 578 et reprise par Takamatsu Corporation en 2006, ou Hōshi 法師 créée en $718^{3}$. Au début du $\mathrm{xx}^{\mathrm{e}}$ siècle, par ailleurs, le tissu économique japonais s'est structuré autour des zaibatsu 財閥, grandes holdings à actionnariat essentiellement familial. La dissolution des zaibatsu au lendemain de la Seconde Guerre mondiale n'a pourtant pas, finalement, réduit le poids des entreprises familiales au Japon, y compris parmi les plus grandes, comme nous allons le voir (Kurashina 2003 ; Allouche et al. 2008).

Les résultats de cette contribution permettront de mieux comprendre la perception des entreprises familiales par le marché, et la surévaluation ou la sous-évaluation qui peut leur être attachée. Dans une première partie, nous exposerons le contexte de cette recherche, préciserons quelques définitions fondamentales, et formulerons, sur la base de la littérature, un jeu d'hypothèses. Notre seconde partie testera empiriquement ces hypothèses à l'aide de méthodes statistiques simples, et discutera des résultats obtenus.

2. Cette méthode consiste à constituer un échantillon de paires d'entreprises, familiale et non familiale, de même taille et de même activité pour chaque paire, ce qui permet de neutraliser l'effet taille et l'effet activité sur la performance. Si, sur l'échantillon, on constate que les unes, par exemple les familiales, ont une meilleure performance que les autres, cela ne peut-être dû à la taille ou à l'activité, puisqu'on a neutralisé l'effet de ces deux variables par la constitution de l'échantillon. Cela est donc très certainement dû au caractère familial ou non des entreprises considérées.

3. Concernant Kongō Gumi, parmi de multiples références dans la presse, voir par exemple : http://english.chosun.com/site/data/html_dir/2005/12/15/2005121561009. html ; s'agissant de Hōshi, voir par exemple http://www.henokiens.com/index_hoshi_ gb.php ainsi que leur site : http://www.ho-shi.co.jp/jiten/Houshi_E/home.htm 


\section{Définitions, contexte et hypothèses}

\subsection{La notion d'entreprise familiale}

Précisons d'abord que la définition de l'entreprise familiale ne bénéficie pas d'un consensus au sein de la recherche en sciences sociales. Cela n'est pas sans poser de sérieuses questions, tant théoriques qu'empiriques. Une étude de Westhead et Cowling (1998) montre en effet que suivant sept définitions différentes, les résultats comparatifs de tests entre entreprises familiales et non familiales aboutissent à des conclusions nettement différentes. Toutefois, Villalonga et Amit (2004) remarquent que la plupart des définitions incluent au moins l'une des trois dimensions suivantes : une ou plusieurs familles détiennent une part substantielle du capital, des membres de la famille possèdent un contrôle significatif sur la firme, et des membres de la famille occupent d'importants postes de direction. Nous nous en tiendrons à ces trois caractéristiques, sans retenir les aspects psychologiques ou sociologiques caractérisant la structure familiale, suivant le nombre de ses membres, leur profession (dans l'entreprise ou hors de l'entreprise familiale) ou leur situation géographique par exemple, comme peuvent le faire Parke (2004 : 366) ou Klein (2008).

Parallèlement, la recherche d'une définition universelle en la matière se révèle quelque peu vaine, dans la mesure où le contexte légal et fiscal relatif à la transmission du patrimoine ou à la séparation possible entre droit au dividende et droit de vote des titres varie sensiblement d'un pays à l'autre. Dans le cas du Japon, Kurashina (2003), en accord avec les démarches les plus courantes dans la littérature pour d'autres pays, a fondé sa définition de l'entreprise familiale sur deux critères : (1) la famille constitue l'un des principaux actionnaires, et (2) des membres de la famille sont actifs dans les structures dirigeantes de l'entreprise, direction ou conseil d'administration. D'autres définitions de l'entreprise familiale au Japon sont recensées par Allouche et al. (2008), mais celle de Kurashina est aujourd'hui, à notre connaissance, la plus largement utilisée par les chercheurs.

Pour être plus précis, Kurashina distingue, outre les entreprises non familiales qu'il qualifie de "type A ", trois types d'entreprises familiales :

- les entreprises familiales à fort contrôle (qu'il qualifie de " type B "), dans lesquelles les membres de la famille sont les principaux actionnaires, détiennent des postes clés de direction et sont présents au conseil d'administration ; 
- celles à faible contrôle de "type C ", entreprises dans lesquelles les membres de la famille sont actionnaires majoritaires mais n'occupent pas de hauts postes de direction ;

- et celles de faible contrôle de «type D ", dans lesquelles les membres de la famille occupent des postes clés de direction mais ne sont pas actionnaires majoritaires.

Kurashina détermine ainsi qu'en 2003, année de publication de son ouvrage, 42,68\% des entreprises japonaises cotées étaient des entreprises familiales, soit 1074 entreprises familiales sur les 2616 entreprises cotées alors au Japon (premier et second marché). Parmi ces 1074 entreprises familiales, 925 étaient de type B, 119 de type C et 30 seulement de type D.

\subsection{Quelques résultats antérieurs sur les entreprises familiales}

Sur le plan international, une étude de Faccio et Lang (2002) indique que les entreprises familiales sont le mode d'actionnariat dominant en Europe de l'Ouest, excepté la Grande-Bretagne et l'Irlande. En Allemagne, Andres (2008) trouve que les entreprises familiales sont plus profitables non seulement que les entreprises non familiales, mais également que celles qui sont détenues par des gros actionnaires non familiaux. La profitabilité n'est donc pas corrélée avec la taille mais bien avec la nature des actionnaires principaux. Andres (2008) remarque aussi que la performance est meilleure lorsqu'un membre de la famille fondatrice occupe un poste de direction. Dans la zone asiatique, considérant neuf pays aussi différents que la Corée du Sud, Hong Kong, l'Indonésie, le Japon, la Malaisie, les Philippines, Singapour, Taiwan et la Thaillande, Claessens et al. (2000) signalent qu'environ deux tiers des entreprises sont détenues par des actionnaires familiaux ou individuels. Aux États-Unis, Anderson et Reeb (2003) observent que près de $35 \%$ des entreprises de l'indice S\&P 500 sont familiales, ce qui rejoint les résultats de McConaughy et al. (1998) pour le Canada. Sur la question précise qui intéresse plus particulièrement cette contribution, Favero et al. (2006) pour les entreprises italiennes, et Sraer et Thesmar (2007) pour les entreprises françaises, confirment que les entreprises familiales affichent une meilleure performance boursière que les autres, que ce soit le fondateur, un de ses descendants ou une autre personne qui occupe la fonction de dirigeant. 
Cependant, Villalonga et Amit (2006), en approfondissant la notion d'actionnariat familial aux États-Unis, distinguent les entreprises selon que le fondateur est le dirigeant ou qu'il s'agit de ses descendants. Dans le premier cas seulement, les entreprises affichent de meilleures performances économiques, financières et boursières. Et si l'entreprise a des moyens pour accroître le poids de certains actionnaires (structure pyramidale, participations croisées ou plusieurs catégories d'actions par exemple), ces mécanismes sont associés à des performances moindres de la firme. Parallèlement, Maury (2006) note à propos de l'Europe de l'Ouest qu'une fonction managériale active des membres de la famille fondatrice, dépassant le simple actionnariat, est positive pour la profitabilité de l'entreprise mais pas pour sa valorisation. La perception d'une bonne rentabilité par le marché n'implique alors pas nécessairement des anticipations de croissance du cours plus élevées que pour les autres entreprises. Cela pourrait indiquer une crainte de confiscation à long terme des bénéfices au profit de certains actionnaires dominants. Le fait que Maury (2006) observe une relation non linéaire entre le niveau de contrôle familial et la performance de la firme indique une diminution des profits attendus de l'actionnariat familial lorsque celui-ci atteint des niveaux de contrôle trop élevés.

La séparation de la propriété de l'entreprise et de son contrôle est génératrice de conflits et de coûts potentiels, ainsi que l'ont mis en évidence Berle et Means (1932) puis plus récemment Jensen et Meckling (1976) avec la théorie de l'agence. Cette théorie souligne l'importance des coûts engendrés par l'asymétrie d'information dans la relation de mandat, par laquelle un mandant confie une mission à un mandataire, appelé agent en anglais, sans savoir parfaitement si ce dernier pourra remplir la mission, ni s'il déploiera tous les efforts qu'elle requiert (Jensen \& Meckling 1976). Il convient donc, nous dit cette théorie, de mettre en place des mécanismes coûteux de contrôle et d'incitation du mandataire, appelé " coûts d'agence ». Dans la grande entreprise moderne des $\mathrm{XIX}^{\mathrm{e}}, \mathrm{XX}^{\mathrm{e}}$ et $\mathrm{XXI}^{\mathrm{e}}$ siècles, de type société anonyme ou autres, les actionnaires confient à des dirigeants professionnels salariés la gestion de leur entreprise : les premiers sont les mandants de la théorie de l'agence, les seconds en sont les mandataires.

Plusieurs types de " coûts d'agence " peuvent être évoqués. Celui tout d'abord qui naît de la relation propriétaire-dirigeant. C'est le plus classique des « coûts d'agence ». Dans cette logique, le rapprochement de la situation d'actionnaire et celle de dirigeant, dans le cas notamment des entreprises 
familiales, devrait réduire ces coûts et donc contribuer à une meilleure efficacité de l'entreprise dans ses processus de décision. S'il y a effectivement réduction des " coûts d'agence " au sein des entreprises familiales, la création de valeur devrait y être supérieure (Anderson \& Reeb 2003 ; Jaggi et al. 2009 ; Villalonga \& Amit 2006).

Un second " coût d'agence " est celui qui est susceptible d'intervenir entre bloc(s) majoritaire(s) et bloc(s) minoritaire(s) d'actionnaires. Un certain nombre d'auteurs a souligné que la concentration actionnariale ne constitue pas le sésame de la création de valeur (Grossman \& Hart 1980 ; Demsetz 1983 ; Himmelberg et al. 1999 ; Demsetz \& Villalonga 2001). En effet, alors que le rapprochement des intérêts des actionnaires et de ceux des dirigeants, rendu possible par un actionnariat familial, réduit un type de coût d'agence, le risque que de gros actionnaires familiaux extraient des bénéfices à leur profit et au détriment des petits actionnaires accroît un autre type de " coûts d'agence ». Savoir quel effet domine reste une question en suspens sur le plan théorique.

Par ailleurs, outre la question des coûts, d'autres aspects peuvent intervenir dans la gestion des entreprises. Ainsi, les entreprises familiales bénéficieraient d'un actionnariat dont l'horizon d'investissement serait davantage le long terme, contrairement à celui d'investisseurs purement financiers supposés rechercher une rentabilité à court terme. Nous pouvons également évoquer un système de valeurs plus homogène dans les entreprises familiales, un lien plus profond entre les membres de la famille et l'activité de l'entreprise, ou encore un moindre recours à l'endettement. Quel que soit l'aspect qui prédomine, les entreprises familiales semblent disposer d'atouts dont sont par nature en partie dépourvues les autres entreprises. C'est pourquoi la valorisation des entreprises familiales par le marché devrait témoigner de la prise en compte de tels avantages, financiers, économiques ou organisationnels, et se traduire par des ratios boursiers plus élevés en moyenne.

Sur le marché suisse, Isakov et Weisskopf (2009) notent que la surperformance des entreprises familiales est à nuancer par plusieurs paramètres. Ainsi, les entreprises familiales (à savoir avec un actionnariat familial d'au moins $20 \%$ dans ce cas) dans lesquelles existe un second actionnaire important (privé, institutionnel ou gouvernemental) sont plus profitables que les autres et mieux valorisées sur le plan boursier. Dans cette configuration actionnariale, les " coûts d'agence " semblent alors se voir réduits entre les dirigeants et les actionnaires, mais également entre les actionnaires 
majoritaires et minoritaires, grâce à une limitation à l'extraction privée des bénéfices. Par ailleurs, ils notent qu'aucune surperformance des entreprises familiales n'est constatée lorsque les membres de la famille ne sont que des investisseurs, et non des membres de l'équipe dirigeante. La connaissance interne approfondie de l'entreprise n'est donc présente que lorsqu'il existe une réelle implication des actionnaires familiaux dans la gestion de la firme. Cette étude se limite cependant à deux mesures de la rentabilité (financière d'une part, économique d'autre part) et une seule mesure boursière (ratio Q de Tobin ${ }^{4}$ ), ce qui en réduit quelque peu la portée. C'est pourquoi nous avons souhaité, dans le cas des entreprises japonaises, apporter des éléments plus détaillés quant à la valorisation boursière comparée des entreprises familiales et non familiales.

Plusieurs auteurs ont souligné une performance supérieure, économique, financière, voire boursière, pour les entreprises familiales (Anderson \& Reeb 2003 ; Maury 2006 ; Villalonga \& Amit 2006 ; Saito 2008), ce qui s'explique selon Anderson et Reeb (2003) par une moindre diversification, et donc une plus grande prise de risque que dans les entreprises non familiales. Dans ce cas, l'équilibre rentabilité-risque des entreprises familiales sur le marché des actions se situe à un niveau supérieur pour les entreprises familiales par rapport aux non familiales, sans que cela conduise nécessairement à une valorisation plus élevée.

\subsection{Sur le cas du Japon}

Kurashina (2003) observe que dans 21 des 33 secteurs que distinguent couramment les autorités boursières japonaises, les entreprises familiales affichent une meilleure performance économique et financière que les

4. Le ratio Q de Tobin met en rapport la valeur de marché de l'entreprise (capitalisation boursière + valeur des dettes au bilan ou cotées s'il en existe) et sa valeur comptable (fonds propres au bilan + valeur des dettes au bilan aussi). Une valeur supérieure à 1 indique que le marché anticipe une création de valeur ou un potentiel de création. Ce potentiel est lié à divers facteurs : avantage concurrentiel, innovation technologique, synergies importantes, etc. Plus ce ratio est élevé plus le marché est optimiste, mais parfois trop si l'on est en présence d'une bulle spéculative par exemple. Un ratio inférieur à 1 est assez inquiétant pour l'avenir de la firme, que le marché perçoit de façon pessimiste (baisse du résultat net, de la rentabilité, etc.). 
autres, alors que dans seulement 7 secteurs sur 33 leur performance est moins bonne. Allouche et al. (2008) confirment ces résultats sur la base de la méthode statistique plus fine que constitue le pairage (voir supra note 2). Ces différents travaux, toutefois, n'abordent pas la question de la performance boursière comparée des entreprises familiales et non familiales.

Dans une étude dont le critère d'identification des entreprises familiales cotées est l'actionnariat uniquement, Nguyen (2011) observe, sur le marché japonais également, que sur la période 1996-2003 il existe une corrélation positive et significative au seuil de $99 \%$ entre le contrôle familial et le price-to-book ${ }^{5}$ d'une part, et entre le contrôle familial et la rentabilité économique ${ }^{6} \mathrm{~d}^{\prime}$ autre part. Il remarque en outre que les entreprises familiales affichent un risque spécifique plus élevé, qui se traduit par une volatilité des actions supérieure à celle des entreprises non familiales. Les entreprises à actionnariat concentré semblent ainsi prendre plus de risque, ce qui accroît plus précisément leur risque spécifique. Si l'on se place dans le cadre d'un marché efficient, la rentabilité ajustée par le risque n'est pas différente d'une entreprise à l'autre. Ainsi, les entreprises familiales ne présenteraient pas de valorisation par le marché supérieure aux autres entreprises après ajustement pour le risque, et leurs variations de cours ne seraient pas différentes des autres.

Il nous est apparu intéressant, en complément de ces travaux, de comparer les performances boursières des entreprises familiales et non familiales au Japon, sur la base de la définition de Kurashina, plus contraignante que celle retenue par Nguyen (2011), mais plus en ligne avec les travaux d'autres chercheurs au Japon et dans le monde entier (Allouche et al., 2008). Compte tenu toutefois des résultats quelque peu contrastés qu'offre la littérature, dans le cas de l'Italie (Favero et al. 2006), de la France (Sraer \& Thesmar 2007), plus largement des pays d'Europe occidentale (Maury 2006) et du Japon (Nguyen 2011), nous avons choisi de tester des jeux

5. Rapport entre le cours de Bourse de l'action et la valeur comptable par action (book value) ; plus il est élevé, plus le marché est optimiste quant aux perspectives de développement et de rentabilité de l'entreprise.

6. Rapport entre le résultat d'exploitation de l'entreprise et son actif économique ; il témoigne, s’il est élevé, de la bonne utilisation par l'entreprise de ses moyens de production. 
d'hypothèses alternatives plutôt qu'une ou plusieurs hypothèses. Ainsi, sur la question de la valorisation boursière, nous nous proposons de tester l'une contre l'autre les deux hypothèses opposées suivantes :

H1a : La valorisation par le marché des entreprises familiales est supérieure à celle des entreprises non familiales.

$\mathrm{H} 1 \mathrm{~b}$ : La valorisation par le marché des entreprises familiales n'est pas différente de celle des entreprises non familiales.

Les études antérieures parviennent à des résultats hétérogènes non seulement quant à la valorisation boursière des entreprises familiales et non familiales, mais aussi sur l'évolution du cours des actions. Ainsi, une meilleure performance économique des entreprises familiales peut se traduire par une variation de cours supérieure sur une période de quelques mois, mais s'atténuer rapidement du fait de l'ajustement de la valorisation en fonction du risque. Parallèlement, les entreprises familiales n'affichant pas de performance suffisante peuvent voir leur valorisation par le marché pénalisée en raison d'une prise de risque potentiellement supérieure. C'est pourquoi nous testons la seconde alternative comme suit :

H2a : La variation annuelle des cours des actions des entreprises familiales est supérieure à celle des actions des entreprises non familiales.

$\mathrm{H} 2 \mathrm{~b}$ : La variation annuelle des cours des actions des entreprises familiales n'est pas différente de celle des actions des entreprises non familiales.

La seconde partie de l'article s'attache à tester ces deux ensembles d'hypothèses alternatives.

\section{Analyse empirique et discussion}

\subsection{Méthodologie et données}

La comparaison entre entreprises familiales et non familiales a été réalisée au travers de paires d'entreprises de même taille et de même activité, l'une étant familiale l'autre non familiale, mais toutes étant cotées sur le marché japonais des actions. N'ont été prises en compte ici que les entreprises familiales à fort contrôle, à savoir celles dans lesquelles des membres de la famille occupent des postes de direction ou font partie du conseil d'administration (Board of Directors), et dans lesquelles la famille fait partie 
des actionnaires principaux. Ne retenir que les entreprises familiales à fort contrôle, celle de «type B » suivant la terminologie de Kurashina (2003), que nous comparerons aux entreprises non familiales, permet d'accroître les chances de déceler des résultats différents si ceux-ci existent réellement. En effet, comme l'ont montré Allouche et al. (2008), les performances sont d'autant plus contrastées entre les entreprises familiales et non familiales que le contrôle familial est fort. Ces auteurs toutefois ne travaillaient pas sur la performance boursière, objet de cet article, mais sur des données comptables et des ratios de rentabilité.

Chaque paire comporte des entreprises de même taille à peu de choses près, et appartenant au même secteur industriel selon le code SIC à quatre chiffres utilisé mondialement. La taille est considérée comme étant à peu près la même si le chiffre d'affaires et le nombre de salariés des entreprises ne présentent pas une différence de plus de $20 \%$.

Les tests statistiques ont porté sur une comparaison globale des écarts sur les indicateurs analysés, entre l'ensemble des entreprises familiales et l'ensemble des entreprises non familiales d'une part, sur la comparaison des écarts moyens par paire d'entreprises d'autre part.

Les indicateurs financiers des sociétés étudiées proviennent de la base Worldscope, les données relatives à l'appartenance familiale sont issues des travaux de Kurashina (2003), et les éléments boursiers ont été extraits de la base FactSet ${ }^{7}$. L'échantillon de sociétés comporte 90 paires d'entreprises, analysées sur la période 1995-2009.

\subsection{Résultats et discussion}

\subsubsection{Présentation comparative des données}

Le tableau 1 fournit les valeurs moyennes des paramètres descriptifs des ratios observés, pour les fins d'années fiscales 1995-1996, 1998-1999, 2001-2002, 2004-2005 et 2007-2008. Pour le price-to-book ratio (PBR), nous pouvons constater que les moyennes sont proches entre les entreprises familiales et non familiales, même si ces dernières affichent une

7. www.factset.com 
dispersion un peu plus élevée. Les valeurs du price-earning ratio (PER) ${ }^{8}$ sont nettement plus élevées en moyenne pour les entreprises non familiales, avec une hétérogénéité similaire. Ces observations indiquent que le marché tend à valoriser davantage les entreprises non familiales sur la base de leurs résultats nets, comme le confirme la variation annuelle moyenne des cours des actions, légèrement plus élevée pour ces entreprises. La variation de cours a été calculée entre le début et la fin de l'année fiscale (fin mars) et est exprimée en $\%$.

\section{TABLEAU 1}

Analyse descriptive des indicateurs boursiers

\begin{tabular}{|l|l|l|l|l|l|l|}
\cline { 2 - 7 } \multicolumn{1}{l|}{ Non familiales } & $\begin{array}{l}\text { Var } \\
\text { cours }\end{array}$ & PBR & PER & P/CF & P/CA & $\begin{array}{l}\text { Div/ } \\
\text { cours }\end{array}$ \\
\hline Moyenne & 16,63 & 1,16 & 24,26 & 9,64 & 0,51 & 2,17 \\
\hline Écart-type & 53,52 & 1,57 & 67,67 & 18,75 & 0,74 & 2,39 \\
\hline Moyenne/écart-type $^{*}$ & 0,07 & 0,78 & 0,55 & 0,56 & 0,69 & 0,90 \\
\hline
\end{tabular}

* Moyenne des valeurs historiques de ce ratio, et non le rapport des deux lignes précédentes.

\begin{tabular}{|l|l|l|l|l|l|l|}
\cline { 2 - 7 } \multicolumn{1}{l|}{ Familiales } & $\begin{array}{l}\text { Var } \\
\text { cours }\end{array}$ & PBR & PER & P/CF & P/CA & $\begin{array}{l}\text { Div/ } \\
\text { cours }\end{array}$ \\
\hline Moyenne & 14,98 & 1,13 & 15,04 & $26,11^{(1)}$ & 0,65 & $3,48^{(2)}$ \\
\hline Écart-type & 49,82 & 1,27 & 25,41 & $141,45^{(3)}$ & 0,76 & $9,66^{(4)}$ \\
\hline Moyenne/écart-type* & 0,04 & 0,97 & 0,59 & 0,50 & 0,89 & 1,26 \\
\hline
\end{tabular}

(1) 9,97 sans deux valeurs extrêmes (une en 1995 et une en 2001)

(2) 2,45 sans une valeur extrême en 2007

(3) 20,40 sans deux valeurs extrêmes (une en 1995 et une en 2001)

(4) 2,90 sans une valeur extrême en 2007

* Moyenne des valeurs historiques de ce ratio, et non le rapport des deux lignes précédentes.

8. Price-earning ratio, rapport entre le cours de Bourse de l'action et le résultat net par action de l'entreprise ; plus il est élevé plus le marché valorise fortement l'entreprise par rapport à son dernier résultat net, ce qui est favorable. 
Quant au rapport entre le cours de Bourse de l'action et la capacité d'autofinancement (CAF) par action $\left(\mathrm{P} / \mathrm{CF}^{9}\right)$, l'écart apparent entre les moyennes n'est dû qu’à deux valeurs particulièrement élevées parmi les entreprises familiales et s'avère donc peu significatif. En ôtant ces deux valeurs extrêmes de l'ensemble de l'échantillon, le P/CF moyen ainsi que les autres paramètres statistiques sont similaires entre les deux groupes d'entreprises. Le rapport entre le cours et le chiffre d'affaires $\left(\mathrm{P} / \mathrm{CA}^{10}\right)$ par action indique que la valorisation boursière est sensiblement la même sur la base du chiffre d'affaires des entreprises.

Enfin, le dernier ratio indique le rendement du dividende ${ }^{11}$, exprimé par le rapport entre le dividende par action et le cours boursier. L'ordre de grandeur des résultats indique des valeurs proches de celles des entreprises occidentales, comme l'a déjà noté Nivoix (2005), ce qui n'était pas le cas avant les années 1990, les entreprises japonaises affichant alors un ratio plus faible (Aoki 1991). Si l'on exclut une valeur atypique en 2007, on constate que ce ratio est seulement légèrement supérieur pour les entreprises familiales alors que leur PER s'avère bien inférieur à celui des entreprises non familiales. Cela s'explique par un taux de distribution des dividendes nettement plus faible en moyenne ${ }^{12}$ dans les entreprises familiales (notion de « capital patient »), signe d'une volonté de conserver des fonds afin de s'autofinancer autant que possible. Ce constat est confirmé par les résultats d'Allouche et al. (2008) qui ont mis en évidence un plus faible niveau d'endettement dans les entreprises familiales.

C'est donc bien le ratio PER, le plus suivi de tous sur le plan boursier, qui seul affiche une valorisation plus élevée pour les entreprises non

9. Price to cash flow; comme le PER, plus il est élevé plus il est favorable, et indique ici une valorisation élevée de l'entreprise par le marché par rapport à sa capacité à générer un surplus monétaire annuel lié à son activité.

10. Price to sales; comme le PER, plus il est élevé plus il est favorable, et indique ici une valorisation élevée de l'entreprise par le marché par rapport à sa capacité à générer un volume de ventes annuelles.

11. Rapport entre le dividende par action et le cours de l'action (dividend yield) ; plus il est élevé plus l'entreprise verse des dividendes importants à ses actionnaires par rapport à la valeur de son action.

12. On peut en effet écrire : Rendement du dividende = Taux de distribution / PER, à savoir Div/Cours = $($ Div/BPA $) /($ Cours /BPA $)$ avec BPA le bénéfice par action. 


\section{GRAPHIQUE 1}

Comparatif des principaux ratios boursiers

pour les entreprises familiales et non familiales

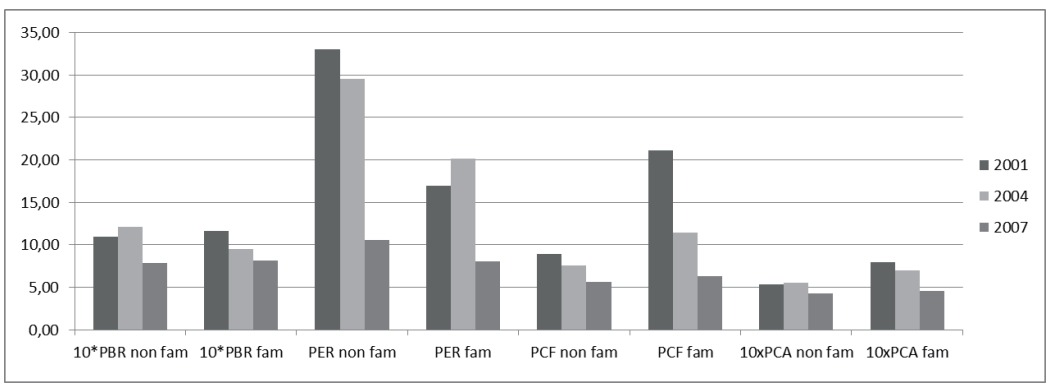

familiales. Il ne s'agit pas ici d'une sous-évaluation des entreprises familiales en raison de leur taille, car ce paramètre a été contrôlé lors de la constitution de l'échantillon à partir de paires. On peut alors proposer une explication en termes de contrôle du capital : les entreprises familiales à fort contrôle apparaissent moins susceptibles d'être cibles d'OPA (offres publiques d'achat) que les autres, et donc moins attractives pour de gros investisseurs. Le contrôle de la gestion par le marché y est également moins présent que dans les entreprises non familiales, dans la mesure où les dirigeants en place sont difficilement remplaçables suite à un vote contraire au cours de l'assemblée générale des actionnaires. On peut alors craindre une gestion sous-optimale de la firme, de possibles "problèmes d'agence » et les coûts qui en découlent, ainsi qu'une tendance à l'enracinement des dirigeants (effet qualifié de control discount dans la littérature anglo-saxonne).

Le graphique 1 indique l'évolution des ratios boursiers pour les trois années fiscales où la totalité des données est disponible. Pour des raisons de lisibilité, les valeurs des ratios PBR et P/CA ont été multipliées par 10. La tendance à la baisse de chaque ratio sur les trois années observées ici est liée à une prudence plus marquée du marché dans ses évaluations postérieures à l'éclatement de la bulle internet, ainsi qu'à une conjoncture économique peu propice à l'optimisme des investisseurs. Et mise à part une valeur atypique qui biaise à la hausse le P/CF des entreprises familiales en 2001, 
nous pouvons remarquer que les différences les plus marquées concernent le PER, plus élevé pour les entreprises non familiales. Cette observation indique une propension du marché à un optimisme plus marqué vis-àvis des perspectives d'activité des entreprises non familiales, ou comme dit précédemment une possible sous-évaluation des entreprises familiales en raison des faibles possibilités d'OPA et de contrôle de leur gestion de façon externe via le marché.

Il convient de vérifier toutefois si les différences entre entreprises familiales et non familiales sont statistiquement significatives, c'est-à-dire si l'on peut raisonnablement considérer qu'elles résultent de différences effectives au niveau de l'ensemble de la population des entreprises, et non du simple hasard d'échantillonnage. Nous avons procédé à cette fin par tests de comparaison de moyennes pour chacun des indicateurs considérés.

\subsubsection{Test de signification des différences constatées}

Nous avons, pour chaque année considérée, analysé statistiquement les écarts pour chacun des indicateurs boursiers précédents entre les entreprises familiales et non familiales. La méthode utilisée, tout à fait classique dans ce genre de démarche, est celle des tests de comparaisons de moyennes des indicateurs considérés. Le test détermine dans quelle mesure les deux sous-échantillons sont susceptibles d'appartenir à deux populations sousjacentes de même moyenne. Dans ce dernier cas, les différences constatées sur les deux sous-échantillons ne seraient pas dues à des différences effectives dans les deux ensembles d'entreprises, familiales et non familiales, mais seraient simplement dues au hasard d'échantillonnage. Ce qui nous permet par exemple de savoir si c'est $\mathrm{H} 1 \mathrm{a}$ ou H1b qui doit être rejetée. Nous pouvons ainsi déterminer si entreprises familiales et non familiales enregistrent effectivement des performances différentes, ou si au contraire les différences constatées sur l'échantillon ne sont dues qu'au hasard de l'échantillonnage ${ }^{13}$.

L'évaluation par le marché se traduit par des écarts de signe variable sur le PBR, et des niveaux de PER plus élevés pour les entreprises non familiales,

13. Les tableaux qui détaillent les calculs ne sont pas fournis ici faute de place, mais seront expédiés par les auteurs à tout lecteur qui en ferait la demande. 
mais statistiquement non significatifs au seuil de $5 \%$ (ce qui signifie que l'on a plus de $5 \%$ de chances de se tromper en prétendant que la différence constatée ici à partir des deux sous-échantillons résulte effectivement d'un différence entre l'ensemble des entreprises familiales et des non familiales). En outre, une tendance des entreprises familiales à afficher de plus forts $\mathrm{P} / \mathrm{CF}$ et $\mathrm{P} / \mathrm{CA}$, ainsi que des dividendes plus élevés, est confirmée, mais, là encore, à des niveaux statistiquement non significatifs au seuil d'erreur de $5 \%$. En d'autres termes, nous prendrions un risque élevé (supérieur à $5 \%$ ) de nous tromper si nous affirmions qu'il y a une différence entre l'ensemble des entreprises familiales et l'ensemble des entreprises non familiales pour les indicateurs considérés. H1a est donc rejetée au profit de $\mathrm{H} 1 \mathrm{~b}$ selon laquelle, rappelons le, la valorisation par le marché des entreprises familiales n'est pas différente de celle des entreprises non familiales.

La variation de cours permet de constater dans quelle mesure l'appréciation de la valeur de chaque entreprise de la paire est identique ou non durant la même période de temps. Les entreprises de chaque paire appartenant au même secteur, l'effet structurel ou conjoncturel lié au domaine d'activité est neutralisé. Nous constatons que les entreprises familiales ont tendance à enregistrer des hausses annuelles de cours moins importantes que les entreprises non familiales, mais de façon non significative au seuil d'erreur de $5 \%$. Dans la même logique que précédemment, cela nous conduit à rejeter $\mathrm{H} 2 \mathrm{a}$ au profit de $\mathrm{H} 2 \mathrm{~b}$, selon laquelle, rappelons-le, la variation annuelle des cours des actions des entreprises familiales n'est pas différente de celle des actions des entreprises non familiales.

\section{Conclusion}

Le marché, malgré des écarts sur les PER, ne semble pas marquer de forte différence dans son évaluation entre les entreprises familiales et non familiales. Il tend même à mieux valoriser les entreprises non familiales, probablement pour les raisons que nous avons signalées, à savoir qu'elles sont moins susceptibles de faire l'objet d'offres publiques d'achat (OPA), ce qui limite les potentialités de gain pour les détenteurs d'action, et parce que les actionnaires peuvent craindre un enracinement plus fort des dirigeants dans les entreprises familiales. 
Soulignons que nos résultats vont dans le même sens que ceux de Nguyen (2011), pour l'indicateur PBR qu'il calcule également. Cet auteur ne calcule pas les autres variables considérées ici, car sa recherche s'inscrit dans une optique d'étude du risque. En revanche, concernant la performance boursière (mesurée ici par la variation de cours) nos résultats diffèrent de ceux de Favero et al. (2006) pour l'Italie et de Sraer et Thesmar (2007) pour la France. Ceci peut provenir de plusieurs causes. Tout d'abord des différences institutionnelles et dans la structure de l'actionnariat dans ces différents pays sont susceptibles d'influencer les performances boursières des entreprises (Davis et al. 1997). Or, les marchés financiers japonais offrent sur la période considérée certaines spécificités : poids relativement faible, quoique croissant, des investisseurs étrangers, niveau encore élevé, même si celui-ci a baissé au cours des deux dernières décennies, des participations croisées, liquidité relativement importante du marché, et exposition comparativement élevée aux activités cycliques. Nous ne pouvons non plus exclure l'influence possible de différences de méthode, de périodes d'observation, de tailles d'échantillon, voire bien entendu de critères de distinction entre entreprises familiales et non familiales. C'est pourquoi nous appelons de nos vœux des travaux comparatifs entre pays utilisant une méthode plus homogène.

Un autre prolongement de notre recherche permettrait d'analyser en détail le niveau de risque de chaque entreprise de chaque paire, afin de déterminer si l'absence de différence de valorisation par le marché correspond bien ou non à un niveau de risque spécifique semblable entre les entreprises familiales et les autres. 


\section{Bibliographie}

\author{
ABDELLATIF Mahamat, AMANN \\ Bruno, JAUSSAUD Jacques, 2010 \\ «Stratégie internationale des \\ entreprises japonaises : étude de \\ l'incidence potentielle du caractère \\ familial », Ebisu, 43 : 29-53.
}

ALLOUCHE José, AMANN Bruno, JAUSSAUD Jacques, KURASHINA Toshiki, 2008

«The Impact of Family Control on the Performance and Financial Characteristics of Family Versus Non Family Business in Japan: A MatchedPair Investigation», Family Business Review, 21 (4) : 315-329.

\section{ANDERSON Ronald \& REEB}

David, 2003

«Founding-Family Ownership and Firm Performance: Evidence from the S\&P 500 », Journal of Finance, $58: 1301-1327$.

ANDRES Christian, 2008 « Large Shareholders and Firm Performance: An Empirical Examination of Founding-Family Ownership », Journal of Corporate Finance, $14: 431-445$.

\section{AOKI Masahiko, 1991}

Économie japonaise, information, motivations et marchandage, Paris, Economica.

\section{AYRANCI Evren, 2010}

« Family Involvement in an Institutionalization of Family
Businesses: A Research », Business

and Economic Horizon, 3 (3) : 83-104.

BERLE Adolph

\& MEANS Gardiner, 1932

The Modern Corporation and Private

Property, New York, MacMillan.

CLAESSENS Stijn, DJANKOV Simeon, LANG Larry, 2000

"The Separation of Ownership and Control in East Asian Corporations ", Journal of Financial Economics,

$58: 81-112$.

DAVIS James, SCHOORMAN David, DONALDSON Lex, 1997

«Towards a Stewardship Theory

of Management ", Academy of

Management Review, 22 (2) : 20-47.

DEMSETZ Harold, 1983

«The Structure of Ownership and

Theory of the Firm », Journal of Law and Economics, 26 : 375-390.

DEMSETZ Harold

\& VILLALONGA Belen, 2001

«Ownership Structure and Corporate Performance », Journal of Corporate

Finance, 7 : 209-233.

FACCIO Mara

\& LANG Larry, 2002

«The Ultimate Ownership of Western

European Corporations ", Journal of

Financial Economics, 65 : 365-395. 
FAVERO Carlo, GIGLIO Stefano, HONORATI Maddalena,

PANUNZI Fosto, 2006

«The Performance of Italian Family

Firms », CEPR Working Paper, $n^{\circ} 5786$.

GEDAJLOVIC Eric, CARNEY Michael, CHRISMAN James, KELLERMANNS

Frantz, 2011

«The Adolescence of Family Firm

Research: Taking Stock and Planning for

the Future », February 1. En ligne : http://

ssrn.com/abstract $=1837578$

\section{GROSSMAN Sanford \& HART}

Oliver, 1980

«Takeover Bids, the Free-Rider Problem, and the Theory of the Corporation ",

Bell Journal of Economics, $11: 42-64$.

HIMMELBERG Charles, HUBBARD

Glenn, PALIA Darius, 1999

«Understanding the Determinants of

Managerial Ownership and the Link

Between Ownership and Performance", Journal of Financial Economics,

$53: 353-384$.

\section{ISAKOV Dušan \& WEISSKOPF}

Jean-Philippe, 2009

«Family Ownership, Multiple

Blockholders and Firm Performance ", Paris, December, Finance International Meeting AFFI - EUROFIDAI. En ligne : http://ssrn.com/abstract $=1484574$

\section{JAGGI Bikki, LEUNG Sidney,}

GUL Ferdinand, 2009

« Family Control, Board Independence and Earnings Management. Evidence
Based on Hong Kong Firms ", Journal of Accounting and Public Policy, 28 (4) : 281-300.

JENSEN Michael

\& MECKLING William, 1976

«Theory of the Firm: Managerial

Behavior, Agency Costs and Ownership

Structure », Journal of Financial

Economics, 3 : 305-360.

KLEIN Sabine, 2008

"Commentary and Extension:

Moderating the Outcome of Family

Confirmation in Family Firms ",

Entrepreneurship, Theory \& Practice,

November : 1083-1088.

KURASHINA Toshiki 倉科敏材, 2003

Famirī kigyō no keieigaku ファミリー企業の

経営学 (Études sur le management des

entreprises familiales ), Tokyo,

Tōyō keizai 東洋経済.

MAURY Benjamin, 2006

«Family Ownership and Firm

Performance: Empirical Evidence from

Western European Corporations ", Journal of Corporate Finance,

$12: 321-341$.

MCCONAUGHY Daniel, WALKER Michael, HENDERSON Glenn, MISHRA Chandra, 1998

«Founding Family Controlled Firms:

Efficiency and Value », Review of

Financial Economics, 7 : 1-19.

NGUYEN Pascal, 2011

«Corporate Governance and Risk-

Taking: Evidence from Japanese Firms ", 
Pacific-Basin Finance Journal, $19: 278-297$.

\section{NIVOIX Sophie, 2005}

« Dividend Pay-Outs and Leverage in Japanese Firms », Asian Business and Management, 4 : 185-203.

PARKE Ross, 2004

"Development in the Family ", Annual Review of Psychology, 5 : 365-399.

\section{SAITO Takuji, 2008}

«Family Firms and Firm Performance: Evidence from Japan ", Journal of the Japanese and International Economies, 22 : 620-646.

\section{SRAER David}

\& THESMAR David, 2007

«Performance and Behavior of Family Firms: Evidence from the French Stock Market ", Journal of the European Economic Association, 5 : 709-751.

\section{VILLALONGA Belén}

\section{\& AMIT Raphael, 2006}

"How Do Family Ownership, Control and Management Affect Firm Value? ", Journal of Financial Economics, $80: 385-417$.

\section{WESTHEAD Paul}

\section{\& COWLING Marc, 1998}

«Family Firm Research: The Need for a Methodological Rethink », Entrepreneurship Theory and Practice, 23 (1) : 31-56. 